\title{
Meeting report British Cancer Research Meeting, 30 June-3 July 2002, Glasgow
} Alicia T Parkes and Valerie Speirs

Molecular Medicine Unit, University of Leeds, Leeds LS9 7TF, UK

Correspondence: Valerie Speirs, Molecular Medicine Unit, Clinical Sciences Building, St James's University Hospital, Leeds LS9 7TF, UK. Tel: +44 (0)1132 065261; fax: +44 (0)1132 444465; e-mail: v.speirs@leeds.ac.uk

Received: 12 July 2002

Accepted: 23 July 2002

Published: 29 July 2002
Breast Cancer Res 2002, 4:202-204

(C) 2002 BioMed Central Ltd

(Print ISSN 1465-5411; Online ISSN 1465-542X)

\begin{abstract}
The 2002 British Cancer Research Meeting was held from 30th June to 3rd July in Glasgow, UK. The meeting was structured to include educational workshops, plenary lectures, symposia, and poster sessions, which brought together scientists and clinicians. Presentations ranged from the impact that modifications to basic chromatin structure can have on diagnosis and targeted gene therapy, to the outcome of novel therapeutics through clinical trials. The emphasis was clear: patient survival is the main priority and treatment of organ-specific cancer must inevitably be replaced by individualised tumour-specific therapy.
\end{abstract}

Keywords: chromatin remodelling, microarrays, targeted therapy

\section{Introduction}

Rain and the city of Glasgow are almost synonymous and sadly this was indeed the case for the duration of the British Cancer Research Meeting 2002, held at the Scottish Exhibition and Conference Centre from 30th June to 3rd July. The rain did not dampen the spirits of the almost 600 delegates, however, and a feast of good science was enjoyed. This ranged from plenary sessions and award lectures to proffered paper sessions and posters, covering various aspects of clinically diagnosed and experimental cancer models. Here we present some of the highlights.

\section{Meeting report}

A number of scientists, both young and established, were honoured at the meeting for their contributions to cancer research. The British Association for Cancer Research (BACR) Tom Connors Award Lecture "Close Encounters of a Molecular Kind" was given by Malcolm Stevens (University of Nottingham, UK). In his lecture, he outlined the need to nurture 'cunning' rather than 'stunning' chemistry as the way forward in drug discovery. The BACR Translational Research Award went to his colleague Tracey Bradshaw for her work on Phortress. In an eloquent presentation, the history of Phortress was outlined, underlining the interplay between chemistry and biology that led to its development and selection as a candidate therapy. Phortress evolved from polyhydroxylated 2-phenylbenzothiazoles designed as potential tyrosine kinase inhibitors. In laboratory studies it has shown very promising results in treating breast and ovarian cancers and it will shortly be undergoing Phase I clinical trials. Marie Boyd (University of Glasgow, UK) received the BACR Young Scientist Award and outlined the exciting prospect of linking conventional radiation-based therapies with gene therapy for treating malignancy.

In "Re-designing Cancer Therapy", Sir David Lane (University of Dundee, UK) emphasised the need for patient-specific therapy, a consensus that was reiterated throughout the conference. He also discussed the necessity of looking not only at the gene in question, but also at the whole signalling pathway in which it is involved. We were reminded that $24 \%$ of human breast cancers have genetic changes in the $p 53$ tumour suppressor gene and how $p 53$ may be utilised as a target for gene therapy. The role of p53 as a potential therapeutic target was also emphasised by Barbara Foster (Pfizer Global R\&D, Groton, CT, USA). Current strategies include the pharmacological rescue of mutant p53 protein, using small chaperone 
molecules. Both speakers discussed various therapeutic strategies for tumours that have mutations in the $p 53$ gene and left us with the notion that the future of $\mathrm{p} 53$ as a therapeutic target was very much alive.

The Cancer Research UK Lecture was given by Olli Kallioniemi (National Genome Research Institute, Bethesda, MD, USA), who discussed the application of array technology in cancer research. From the construction of simple RNA or DNA arrays, this field has evolved rapidly in recent years. The emerging challenge now is to uncover the function of the many candidate genes identified in earlier genome arrays by integrating gene copy number and gene expression analysis with functional studies. The development of tissue microarrays, in which it is theoretically possible to represent an entire pathology archive on a single microscope slide ('pathomics'), is one way forward for such high-throughput analysis. Tissue microarrays allow population-level screening and cellular resolution, which are not possible using genome arrays. A novel slant is the use of living gene transfection microarrays [1]. With this technique, cultured cells are overlaid on DNA arrays. Those cells that take up DNA create spots of localised transfection. This exciting new technology has applicability for identifying new drug targets and in discovering gene products which affect cell physiology.

Another innovative angle involves harnessing the power of gene transfection microarrays with RNA interference [2]. RNA interference involves the processing of dsRNA to short interfering RNA of about 21 nucleotides, as a form of post-transcriptional gene silencing. This is often manifested in an aberrant phenotype, which can allow gene function to be identified. Quite literally we now have available an array of arrays and, bearing in mind how rapidly this field continues to evolve, the 'laboratory-on-a-chip' may soon be here.

The importance of histone modification and chromatin remodelling is becoming increasingly recognised, particularly since many important genes are methylated and epigenetically silenced in tumours. In a whole session dedicated to this subject, the audience was educated on a range of topics, from basic principles of chromatin structure, to the role of histone modifying proteins and methylation in chromatin remodelling. Histone deacetylases and DNA methyltransferase inhibitors could represent new diagnostic or therapeutic targets. Tony Kouzarides (Wellcome/Cancer Research UK Institute, Cambridge, UK) initiated this session in the BACR Frank Rose Lecture, giving an invigorating and informative insight into the role of "Histone Modifications in Transcriptional Control". Particular emphasis was given to the effect of gene methylation on transcriptional repression, and he gave an illustrative demonstration of the downstream effects of these events. Interestingly, he used the estrogen receptor (ER)-regulated gene $p S 2$ as a model to demonstrate the complex sequence of events regulating gene transcription. The focus of the talk was on lysine and arginine methylation. Using chromatin immunoprecipitations, he showed that methylation of arginine 17 on the histone $(\mathrm{H} 3)$ tail is required for activation of the $p S 2$ gene. The order of events involves recruitment of histonemodifying proteins, acetylation, and subsequent methylation of the gene before its activation. The presentation was concluded with the possibility of using this detailed information on $p S 2$ to produce better defined targets for therapy and molecular diagnosis.

Individual tumours have different patterns of methylation, and Robert Brown (University of Glasgow, UK) also emphasised the potential of identifying gene methylation patterns in specific tumours. Nick La Thangue (University of Glasgow, UK) highlighted the use of histone deacetylase inhibitors as cancer therapeutics. One candidate, PXD101, is a novel histone-deacetylase antagonist that blocks tumour growth by inducing apoptosis and has little toxicity in mice. Advances in cancer therapeutics are continually ongoing, and novel strategies using DNA methyltransferase inhibitors and histone-deacetylase inhibitors are already showing promise in clinical trials.

New predictive markers for breast cancer are always being sought and work presented by Ramsey Cutress (University of Southampton, UK) showed that BAG-1, a multifunctional protein with targets including heat shock proteins and some nuclear hormone receptors, may have potential in predicting response to hormonal therapy. In a study of 138 breast cancers, nuclear expression of BAG-1 was associated with improved survival. BAG-1 interacts directly with ER- $\alpha$ and - $\beta$. Brid Ryan (St Vincent's University Hospital, Dublin, Eire) showed that survivin, an inhibitor-of-apoptosis protein, may have potential in prognosis. Survivin is overexpressed in many tumours, including breast, is inversely correlated with expression of ER and progesterone receptor and is positively correlated with tumour grade.

Current indicators of response to breast cancer therapy rely on the hormonal status of tumour tissue. Tumours responsive to endocrine hormones are treated with the ER antagonist tamoxifen. The therapeutic efficacy of tamoxifen is compromised by its agonistic activity in some tissue types. Mitch Dowsett (Royal Marsden NHS Trust, London, UK) highlighted that ER-negative (ER-)/progesterone receptor-positive patients also benefit from hormonal therapy and suggested that identification of progesterone receptor in the small subset of ER- tumours (25\%) should also be ascertained prior to selecting adjuvant therapy. He described the reduced benefit tamoxifen might have on patients that have $\mathrm{ER}^{+}$and HER-2 ${ }^{+}$tumours.

Matti Aapro (Clinique de Genolier, Switzerland) addressed a number of consensus guidelines for adjuvant therapy, 
and both Aapro and Dowsett summarised the findings of the recent ATAC trial in which the aromatase inhibitor anastrozole was found to be superior to tamoxifen or a combination of both drugs in treating postmenopausal women with early breast cancer [3]. Aapro proposed the use of adjuvant drugs such as Paclitaxel, in the early treatment of breast cancer, to improve patient survival. He concluded that one specific drug treatment will not prove successful for all patients and that organ-specific cancer treatment will soon be superseded by tumour-specific therapy. This idea was reinforced by Dowsett, who encouraged the idea that tumours should be seen as individual entities and should be treated specifically.

Tumour metastases and drug resistance remains a burden on the current treatment of breast cancer. Both Graham Roche-Nagle (Beaumont Hospital, Dublin, Eire) and Thomas Barry (Mater Hospital, Dublin, Eire) presented data illustrating the role that cyclo-oxygenase-2 (COX-2) may play in these events. Using selective COX-2 inhibition Roche-Nagle demonstrated a significant reduction in the number of tumour metastases in an orthotopic murine model of breast cancer. Microvessel density and levels of serum vascular endothelial-cell growth factor were also reduced by COX-2 inhibition.

Drug delivery and targeting are important aspects of cancer therapy that were covered in several presentations. The story of 5-(aziridin-l-yl)-2,4-dinitrobenzamide (CB1954) was presented by Richard Knox (Enact Pharma plc, Salisbury, UK). CB1954, developed in the 1950s, is a proven success in eradicating rat tumours, but unfortunately the same effect was never observed in human cancers. This is due to differences in the bioactivation of CB1954 in rats versus humans: the human form of the enzyme DTdiaphorase (NQ01) is much less efficient at metabolising CB1954, which accounts for its lack of activity against human cancers. New research has identified an enzyme (NQ02) in some human tumours, although not those of the breast. NQ02 is several-fold more effective than NQ01 at metabolising CB1954 and a Phase I trial is about to begin, with the aim of establishing the efficacy of NQ02 as a more active substrate for CB1954.

Ruth Duncan (University of Cardiff, UK) presented an overview of tumour-specific drug delivery using polymer-anticancer conjugates. To date, successful receptor-mediated targeting has been limited to the asialoglycoprotein receptor in hepatic carcinomas, but HER2/neu, present in some breast cancers, could represent a new target for polymer-based drug delivery.

Gerry Potter (De Montfort University, Leicester, UK) described some of the actions of the human cytochrome $\mathrm{P}_{450}$ CYP1B1, the overexpression of which has been cytochrome $\mathrm{P}_{450}$ enzymes are known to metabolise a variety of anticancer drugs; the consequence of cytochrome $\mathrm{P}_{450}$ metabolism is usually detoxification of the drug, although bioactivation occurs in some cases. With this in mind he proposed that CYP1B1 be reclassified as a tumour-suppressor enzyme, through natural prodrug bioactivation. He presented data showing that some natural dietary agents, such as phytoestrogens and resveratrol, are converted into anticancer agents by CYP1B1.

\section{Conclusion}

As with most international conferences, the gathering of many researchers, scientists and clinicians results in the generation of novel ideas and extensive discussions. The outcome, foremost, drives delegates forward in their areas of cancer research. As the ultimate focus is on improving patient survival using chemopreventative and chemotherapeutic drugs, we are reminded of the demands of the patient. Perhaps the most significant message from this meeting is the necessity and inevitable development of a tumour-specific drug that is tailored to each patient's gene profile, thus meeting their individual requirements. This is keenly awaited. Perhaps the results of research in these areas, including interim results from the Phase I trials mentioned above, will be presented at the next meeting. The next British Cancer Research Meeting will take place at the Bournemouth International Conference Centre on 2-5 July 2003 (http://www.icr.ac.uk/bacr/home.htm).

\section{Acknowledgements}

The authors' attendance at this meeting was made possible by the provision of a BACR student travel bursary to ATP and a Yorkshire Cancer Research travel grant to VS. We thank these organisations.

\section{References}

1. Ziauddin J, Sabatini DM: Microarrays of cells expressing defined cDNAs. Nature 2001, 411:107-110.

2. Bass BL: RNA interference: The short answer. Nature 2001, 411:428-429.

3. The ATAC (Arimidex, Tamoxifen Alone or in Combination) Trialists' Group: Anastrozole alone or in combination with tamoxifen versus tamoxifen alone for adjuvant treatment of postmenopausal women with early breast cancer: first results of the ATAC randomised trial. Lancet 2002, 359:2131-2139. 